Outcome of the ARAMIS Project: Accidental Risk Assessment Methodology for Industries in the Framework of the SEVESO II Directive - New Stakes and Opportunities in the Control of Major Accident Hazards in Europe: Outputs from the ARAMIS Project

doi:10.1016/j.jhazmat.2005.07.013

Copyright (c) 2005 Elsevier B.V. All rights reserved.

\title{
The use of generic failure frequencies in QRA: The quality and use of failure frequencies and how to bring them up- to-date
}

\section{H.I. Beerens*, J.G. Post and P.A.M. Uijt de Haag}

RIVM, P.O. Box 1, 3720 BA Bilthoven, The Netherlands

* Corresponding author. Tel.: +3130 2743618; fax: +31302744442.

\begin{abstract}
Quantitative Risk Assessment (QRA) is a method which is often used in the chemical industry and, in some countries, also in land-use planning. In QRA calculations the frequency of an accident scenario is most often assessed by a generic failure frequency approach. The credibility and validity of the failure frequencies used in the Netherlands for land-use planning is evaluated by means of an historical review. Furthermore, the possibility is presented how these generic data can be revised and updated.
\end{abstract}

\section{Introduction}

Risk assessment is of great importance in the (petro-) chemical industry. Although safety standards are high in this industry, one incident can have a tremendous impact on the environment due to the flammable or toxic nature of the chemicals which are processed and stored. To maintain or improve the safety and to make cost effective choices it is essential to know what the risk causing factors are. For this purpose, risk assessment is an excellent tool.

One method widely used in chemical industry is quantitative risk assessment (QRA) which produces quantitative results for the risk of a chemical installation (process, storage, transport). The QRA method allows adding up the risk of all installations to the total risk of a site. Moreover, this method also makes it possible to distinguish the risk contributions of various parts of an installation. In this method, scenarios and the corresponding failure frequencies play a significant role.

The accuracy of the calculations is determined by the quality of the data used. To use QRA methods for land-use planning, it is of great importance that the results are standardized using reliable data. However, the set of available failure frequencies is nowadays not up-to-date anymore, not only in the Netherlands but all over Europe and in fact worldwide. Thus, there is a necessity for reviewing and updating the failure frequencies defined in guidebooks, like the Purple Book [1], for a number of standard scenarios and installations. 
In this article, different methods of risk assessment are outlined as well as the use of failure frequencies in these methods. The reliability of the failure frequencies used nowadays is described using the scenarios for pressure vessels as an example. Finally, the present generic failure frequencies are discussed as well as the possibility to update and improve them.

\section{Methods of risk assessment}

There are many methods for risk assessment known in literature and used in practice [2]. Some of them, like Hazard and Operability Study (HAZOP) and Failure Mode and Effect Analysis (FMEA) are used to identify the risk of an installation and its process. Once the risk is known appropriate steps can be taken to improve the safety. Although these methods are very detailed for a specific installation, they are not quantitative and not suited to rank the contribution of specific installations to the total risk of a chemical plant. The strength of HAZOP and FMEA is identifying possible hazards when e.g. an installation or the process conditions are changed.

In case of a QRA, on the other hand, it is possible to quantify the risk of an installation. The QRA method is developed and in use from the early eighties of last century [3] and [4]. In a QRA, accident scenarios are used. In Fig. 1, this is schematically presented in the so-called bow tie. The figure shows that the scenario starts with a basic failure event and leads to the central event (the accident, e.g. the release of a chemical substance). Subsequently from this central event several and different consequences can be developed with their specific outcomes.

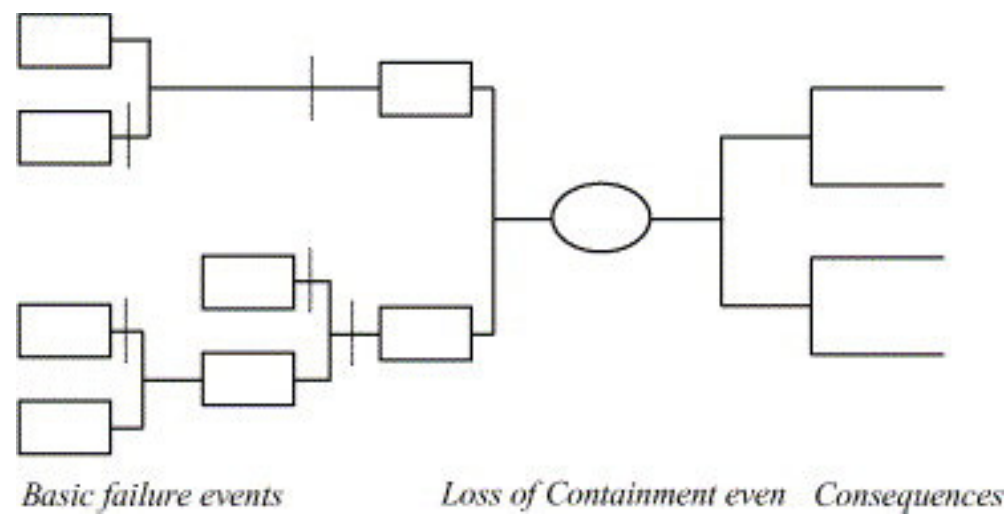

Fig. 1. Bow tie representing the basic failure events, the Loss of Containment accident and the consequences. Barriers preventing the accident are indicated with dashed lines.

There are several levels of detail in which a quantitative risk assessment can be carried out. In the nuclear industry, the so-called probabilistic safety assessment (PSA) is generally used. In this method, the possibility of having an accidental failure is developed from the failure frequencies of the basic system components like pumps, pressure sensors and level gauges. This PSA gives much insight in the different contributions of all the components in an installation to the resulting risk. However, the method is very elaborate and requires much credible data for reliability and failure of these components.

In the chemical industry a QRA, in general, is less detailed. Failure frequencies are only used for the main components or even only for the accidental event. The 
frequencies currently used are based on historical data of incidents. Several datasets of failure frequencies exist, like Oreda and Eireda. Off-shore Reliability Data (Oreda) [5] is an ongoing set of reliability and failure frequencies resulting from cooperation of platform operators of the North Sea and Norwegian Atlantic. European Industry Reliability Databank (Eireda) is the same kind of data, mainly of European power generator companies [6].

In the next sections, it is presented how the failure frequency data in the Netherlands are developed in the past 25 years, using pressure vessels failure frequencies as example, and how updating in Europe is anticipated.

\section{History of the pressure vessel failure frequencies in the Purple Book}

This section discusses the historical background of the failure data for pressure vessels in the Purple Book. More specifically, the origin of the failure frequencies is investigated, concentrating on the historical data sources used in the COVO study [3]. Of the six industrial objects analysed in the COVO study, emphasis is given to the Akzo chlorine storage. The historical outline followed in the literature survey is depicted in Fig. 2.

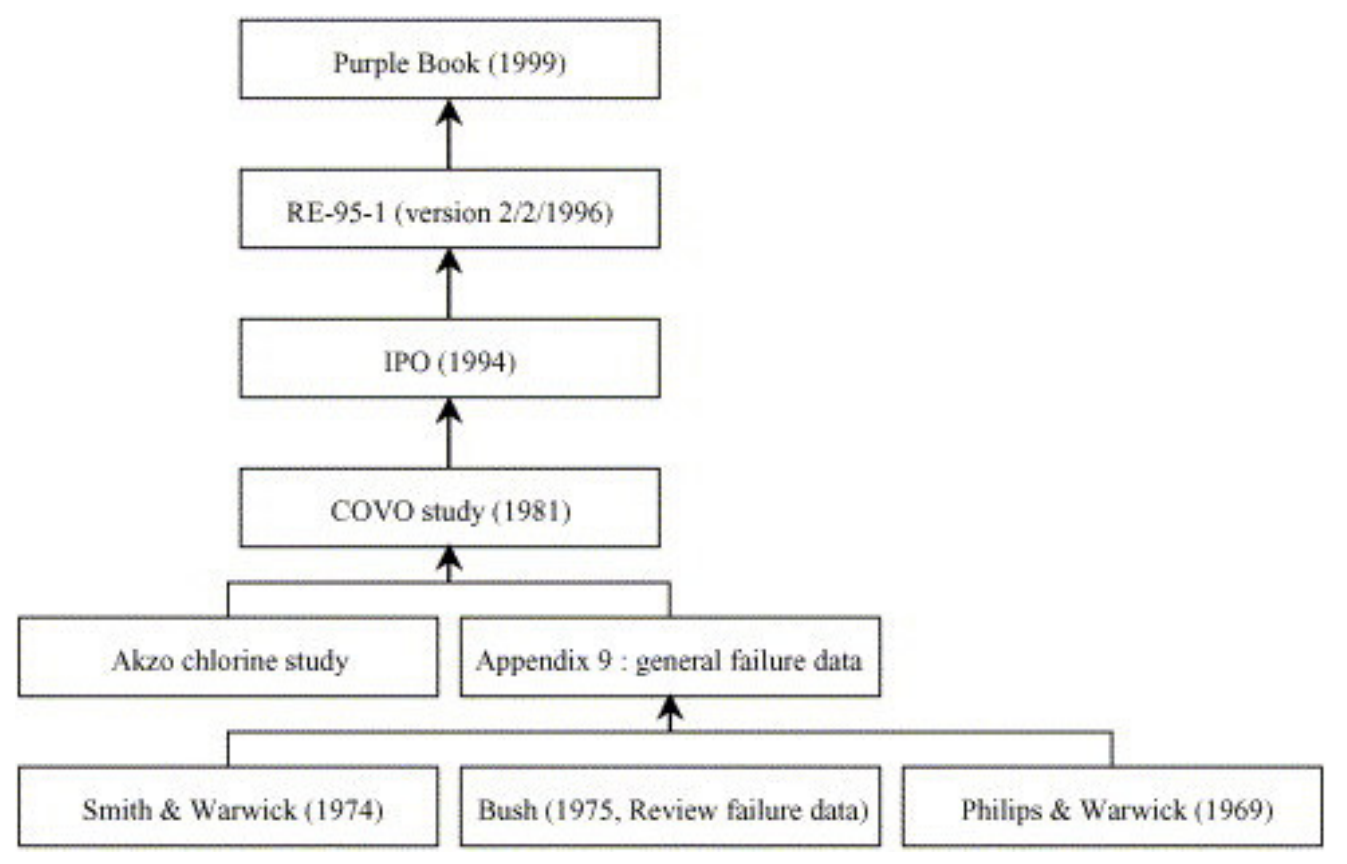

Fig. 2. Historical outline started from the COVO study and the references cited therein.

\subsection{The Covo study (1981)}

Part of the COVO study comprised the study of the Akzo chlorine pressure storage, in which two different approaches were used for the determination of the failure frequencies. The catastrophic failure of a tank was derived from fault-tree analysis, whereas the other failure types (leakages) were based on historical data. The inclusion of failure causes like human error, corrosion and fatigue due to vibrations in the fault-tree complicates the comparison with the Purple Book data, in which these failure types are excluded. The leakages of vessels and pipelines are based on the historical data, incorporated in Appendix 9 of the COVO study. Only one frequency is given for leakages and is defined as "serious 
leakage" for vessels and "significant leakage" for pipelines. Because of the lack of an exact definition, the failure causes which are included as well as the hole size must be estimated to make a comparison with the Purple Book data possible.

In the references cited in the COVO study, failures for pressure vessels were classified in two categories: potentially and catastrophic failure [7] and [8] and noncritical and (potentially) disruptive failure [9]. Contrarily, for vessels, the COVO study makes a distinction between catastrophic rupture and serious leakage. Although the COVO study refers to these preceding studies, there is no clear link between the different types of classifications. The question posed is to what extent potential failure must be taken into account in a failure frequency study.

Furthermore, the values collected by Philips and Warwick [7], Smith and Warwick [8] and Bush [9] are based mainly on data for steam generation systems and a very limited number of process type vessels. The failures quoted by these UKAEA studies involved only some $10-25 \%$ actual catastrophic rupture of vessels. In order to obtain an estimate of the base failure rate for pressure vessels, in the COVO study the range quoted in the above-mentioned references is divided by a factor 10 . However, there is no good basis given for this conversion of data.

Bush [9] reviews different data sources from various countries. An additional limitation in the examination of these data is the use of upper limits of confidence levels based on zero leakage. As a result of the unclear indication of the confidence level used for certain data, some differences in the reported failure frequencies cannot be explained. Some data sets are basically subsets of larger extrapolated data sets, which as a consequence can not additionally contribute to the determination of an appropriate upper bound value for failure probability. Moreover, these historical data sets do not give enough information for the assignment of a leak to the vessel or to the pipelines connected to the vessels.

In some data sets, failure rates for pressure vessels are included originating from defects found at periodic inspections. One cannot exclude the notion that many of the "failures" cited were probably generated during the fabrication stage and remained virtually unchanged until detected during a periodic inspection. These types of failures did not, however, directly interfere with functional use and do not result in emission. In order to prevent confusion, attention should be given to imprecise definitions and inconsistent use of terms such as "failure statistics" and "catastrophic failure".

\subsection{IPO (1994)}

The first document in which standardised failure frequencies were published by the Dutch authorities was the IPO document [10]. The frequency of catastrophic failure for a pressure vessel in the IPO is in good accordance with the value in the COVO study $\left(10^{-6}\right.$ per year). Catastrophic failure is modelled as instantaneous release $\left(5 \times 10^{-7}\right.$ per year $)$, release in $10 \mathrm{~min}\left(2.5 \times 10^{-7}\right.$ per year $)$, and release through a diameter of $50 \mathrm{~mm}\left(2.5 \times 10^{-7}\right.$ per year $)$. For leakages the comparison is more complicated. Whereas in the COVO study only "serious leakage" $\left(10^{-5}\right.$ per year) is defined, in the IPO-study two types of leakages are considered: leakage from a hole with a diameter of $50 \mathrm{~mm}\left(10^{-5}\right.$ per connection) and leakage from a hole with an effective diameter of $10 \mathrm{~mm}\left(10^{-5}\right.$ per year). In fact, the frequency of $10^{-5}$ per year is transformed into $(n+1) 10^{-5}$ per year, in which $n$ is the number of connections. Another point of discussion is that the serious leakage defined in the COVO study as a leak of $50 \mathrm{~mm}$ on an arbitrary position on the tank, is transformed in the IPO into a leakage from a hole of $50 \mathrm{~mm}$ on the 
Journal of Hazardous Materials Volume 130, Issue 3, 31 March 2006, Pages 265-270

connection of the pipelines. On the other hand, in the COVO study there is a separate scenario defined for the rupture of connections on the tank. 


\subsection{RE-95-1}

The IPO document was amended in the RE-95-1 document [11]. In the IPO document, a leak from a hole with an effective diameter of $50 \mathrm{~mm}$ (with a failure frequency of $10^{-5}$ per year) is defined. This Loss of Containment event (LOC) was meant to cover the rupture of the pipe connected to the vessel; however, rupture of pipes is already covered by the LOCs of pipes. To avoid double counting, this LOC is left out in the RE-95-1. and only the class of leakage with a hole size of $10 \mathrm{~mm}$ is left. One could ask whether this omission can be justified. This consideration is based on the fact that the frequency of $10^{-5}$ per year is considered as the frequency for serious leakage and therefore probably has a hole size larger than $10 \mathrm{~mm}$. As a consequence, the deletion of the $50 \mathrm{~mm}$ category may be an underestimation.

The three scenarios for catastrophic failure, defined in the IPO document, were reduced to two scenarios in the RE-95-1: instantaneous release $\left(5 \times 10^{-7}\right.$ per year) and release of the complete inventory from a hole with an effective diameter of $50 \mathrm{~mm}$ or, if the duration exceeds ten minutes, a continuous release with a duration of ten minutes $\left(5 \times 10^{-7}\right.$ per year $)$.

\subsection{Purple Book (1999)}

The Purple Book is based on the IPO and the RE-95-1. In the RE-95-1, the LOC of $5 \times 10^{-7}$ per year is defined as a release from a hole with an effective diameter of $50 \mathrm{~mm}$ or a continuous release in ten minutes. For simplicity, this LOC is in the Purple Book defined as a release of the complete inventory in ten minutes at a constant rate of release. In other words, the catastrophic failure is defined in the Purple Book as an instantaneous release or a release in ten minutes. The total history of the vessel failure frequencies is depicted in Fig. 3.



Fig. 3. History of vessel failure frequency data. 


\subsection{Conclusions}

This study pointed out that it is rather difficult to judge the reliability of the sources. Both the link between the IPO and the COVO study, as well as the derivation of the failure frequencies from the references of the COVO study, are not evident. This can be attributed to various aspects:

- There is no univocal terminology used for the classification of the failures, which complicates the comparison of the data. Moreover, the expression "catastrophic failure" has different meanings in the different reports, which shows the difficulties in the application of uniform criteria.

- Besides real failure, a lot of potential failure frequency data were considered. Failures during inspection and testing (pressure) are also included. This contradicts the Purple Book, where these failures are not included.

- The information in the data sets is often limited and does not always give an indication of the kind of failure causes included and the confidence upper boundary of the failure numbers. Furthermore, the underlying assumptions and relationships to certain causes are not unambiguously described. Some of the studies are not representative, because they are based on an extremely small data set.

- Rough estimates are made in order to convert data from one type of industry (steam generators), into data for another type of industry.

\section{The current generic data set and the necessity for updates and revision}

\subsection{In the Netherlands}

An important source of uncertainty in the results of risk assessments is the use of different data sets for failure frequencies by risk assessors.

The problem of repeatability in risk assessments can be solved to a certain level by giving a fixed standard value for release frequencies for different types of equipment. In the Netherlands, this is described in the Purple Book, including standard scenarios and failure frequencies. These frequencies are set as default values; it is recognised that these data will only be seen as an approximation for any specific plant. Deviation is possible, but only on the condition that it can be motivated and approved by the competent authorities. Lower frequencies can be used if there are special provisions additional to the standard provisions. Higher frequencies should be used if standard provisions are missing or under uncommon circumstances. In fact, the use of generic data requires a determination of the conditions under which the data would be appropriate, something which is not well-defined to date. As a consequence, data are used outside the context for which they are originally collected.

Besides the uncertainties mentioned in the previous section, another disadvantage of the current set of standard failure frequencies, which should not be underestimated, is the fact that they are based on outdated numbers. Most data were published around 1970, which means that most of the data are at least 25 years old. It could be argued that because of the changes of design rules, materials and experience the failure frequencies have changed since then. However, at the moment there is no real proof of this from the data found in 
literature. It is unknown to what extent these data are representative for the current standard in the chemical industry.

The failure frequencies are based on a limited number of characteristics. For vessels, there is only a distinction between storage vessels and process vessels, and for pipes only the length and the diameter is relevant. As a consequence, special provisions directed to integrity of the installation and significant for safety are not included in the failure numbers. Moreover, review studies have been published that show a tendency for some systems towards higher failure frequencies than the ones reported in the Purple Book [12] and [13]. These figures clearly underline the need to have a better understanding of the actual failure data and the underlying assumptions.

To meet this objective a study to revise and update the standard failure data has been started [14]. One of the goals of the study is to throw some light on the way in which equipment-release frequencies vary according to the standards of design, construction, operations and maintenance and to the actual operating conditions. The intention is also to give some guidance on the way the release and accident frequencies depend on the underlying assumptions.

The approach used involves the selection of baseline frequencies for different equipment types in combination with modification factors. The baseline frequencies can be corrected by these factors to obtain a more realistic risk estimate of the actual frequency. The failure frequencies obtained in this way will be very useful for non-standard applications. When choosing modification factors for release frequencies it is necessary to decide in relation to what the modification is made. A baseline of design, construction, operations and maintenance standards is needed. In order to determine the reference standards, it is necessary to look at the data sources. The main sources of data in this study are of recent date and varied: company data from the oil, petrochemical and chemical industries covering the mid-1970s to the present day are included. As model for failure rate prediction a fault-tree-based model will be used in combination with a checklist-based algorithm. Algorithms will be made only for the causes of failure that arise most frequently. For the modification factors care should be taken that some factors, like special corrosion, are not taken into account twice. More specifically, when a correction is made for the application of the equipment item, it is not always necessary to provide an additional correction for corrosion.

At the time of writing work is still in progress to determine appropriate baseline values, modification factors and to apply the above-mentioned approach [14].

\subsection{In Europe}

In Europe, since the 'Seveso-2' directive [15] came into force, EU-member states are required to develop a land use policy in which their residents are protected against major accidents which can possibly happen at 'Seveso' sites (major chemical installations). To facilitate countries in developing and carrying out this policy the competent authorities of the member states decided to develop or identify appropriate methods and data sets. A Land-Use-Planning project was started for this purpose [16]. One of the first activities in this project was gathering information from EU member states about the use and availability of failure frequencies data in their country. It turned out that in most countries where failure frequencies are used, there is no standard set of up-to-date failure frequencies available. In many cases, the Purple Book or UK HSE data are used. 
Therefore in the Netherlands, as well as in Europe, there is an urgent need for revised failure frequency data.

After many communications with experts both from governmental organisations as well as from industry, it was felt that there is a widely shared need to improve the status of failure frequencies for (on shore) chemical installations. To meet this demand there was a meeting of experts in this field (in March 2005), with the aim of starting the development of an up-to-date failure frequency data set. As a result of this meeting a working group will update the failure frequency data. For this purpose the available 'public' data, as well as 'private' data of the members will be used.

\section{Conclusion}

Quantitative Risk Assessment is widely used for improving safety and for land use planning. For this purpose, there is a strong need for failure data that are actual, reliable and generally accepted.

This study shows the difficulty of tracing back failure data due to the lack of univocal terminology. The failure data of pressure vessels in the Purple Book have been copied from the IPO. The unclear links between the IPO and the COVO study, as well as between the references of the COVO study and the COVO study itself, seriously hamper the validation of failure data in the Purple Book.

To obtain validated failure data we initiated a study based on recent data and a fault-tree-based model with algorithms and modification factors. This initiative should ultimately lead to failure data that can be easily applied in current-day practice and gives opportunity to obtain appropriate data for non-standard applications. This study will be discussed in the meeting of experts which will be organized, in spring 2005, to consider the collective need for an updated failure frequencies data set and to explore the possibilities to achieve this.

\section{References}

[1] Committee for the Prevention of Disasters (CPR), Guidelines for Quantitative Risk Assessment"Purple Book" CPR18E, SDU, The Hague, 1999.

[2] J. Tixier, G. Dusserre, O. Salvi and D. Gaston, Review of 62 risk analysis methodologies of industrial plants, J. Loss Prevention Procc. Ind. 15 (2002), pp. 291-303.

[3] COVO Commission, Risk Analysis of Six Potentially Hazardous Industrial Objects in the Rijnmond Area, A Pilot Study. A Report to the Rijnmond Public Authority, Central Environmental Control Agency, Schiedam, The Netherlands, 1981.

[4] F.P. Lees, Loss Prevention in the Process Industries (second ed.), Butterworth-Heinemann (1996).

[5] Offshore Reliability Data Handbook (Oreda), third ed., Det Norsk Veritas, Høvik, Norway 1997.

[6] H. Procacaccia, S.P. Arsenis and P. Aufort, European Industry Reliability Databank (Eireda) (third ed.), Crete University press, Iraklion, Greece (1998).

[7] C.A.G. Philips, R.G. Warwick, A Survey of Defects in Pressure Vessels Built to High Standards of Construction and its Relevance to Nuclear Primary Circuit Envelopes, Authority Health and Safety Branch, Risley, Warrington, Lancashire, UK, AHSB (S) R162, UKAEA Report, 1969. 
Journal of Hazardous Materials Volume 130, Issue 3 , 31 March 2006, Pages 265-270

[8] T.A. Smith, R.G. Warwick, The Second Survey of Defects in Pressure Vessels Built to High Standards of Construction and its Relevance to Nuclear Primary Circuits, United Kingdom Atomic Energy Authority, Warrington, SRD-R-30, UKAEA Report, 1974.

[9] S.H. Bush, Pressure vessel reliability, J. Pressure Vessel Technol. (1975), pp. 54-69.

[10] IPO, Guidelines for the Preparation of Off-site Safety Industrial Sites, Report IPO Project A-73, The Hague, 1994 (in Dutch).

[11] RE-95-1, Version 2-2-1996, KO-95, KO-96, KO-100 performed by TKO Working Group, 1996.

[12] M.Th. Logtenberg, Derivation of Failure Frequencies for LOC Cases, TNO, Apeldoorn, The Netherlands (1998).

[13] Handboek kanscijfers voor het opstellen van een veiligheidsrapport, Co-ordinated Version 2.0, AMINAL Dienst gevaarlijke stoffen en risicobeheer, Brussels, 2004.

[14] J.R. Taylor, Hazardous materials release and accident frequencies for process plant, Draft version, 2004.

[15] Council Directive 96/82/EC of 9 December 1996 on the control of major-accident hazards involving dangerous substances, Office for official publications of the EC, Luxembourg, 1996.

[16] Website http://landuseplanning.jrc.it. 